\title{
Apoio social e síndrome da fragilidade em idosos residentes na comunidade
}

\author{
Social support and the frailty syndrome \\ among elderly residents in the community
}

Fabienne Louise Juvêncio dos Santos Amaral ${ }^{1}$

Ricardo Oliveira Guerra ${ }^{1}$

Aline Freire Falcão Nascimento ${ }^{1}$

Álvaro Campos Cavalcanti Maciel ${ }^{1}$

Departamento de

Abstract Frailty among the elderly is a result of the complex interplay between social and clinical factors involved in its genesis. However, it is still unclear whether the fraailty syndrome can be aggravated due to lack of social support. The scope of this study was to analyze the association between social support and the frailty syndrome among elderly residents in the community. It is an analytical observational cross-sectional study, with a sample of 300 elderly people. Socio-demographic, economic and physical health, frailty and social support data were collected. For statistical analysis, the Pearson chi-square, the Student $t$ and the binary logistic regression were performed, adopting a significance level of 5\%. No associations were observed between frailty and the social support variables, except for the housekeeping aspect $(p=0.04)$. In logistic regression frailty with age $(O R=1.19)$, physical activity $(O R=2.56)$ and self-perceived health $(O R=3.33)$ remained associated. It is considered that more studies need to be conducted to characterize social vulnerability and health services also need to recognize the importance of social support as an integral part of care for the elderly.

Key words Aging, Frailty among the elderly, Social support
Resumo A fragilidade em idosos resulta da complexa interação entre fatores clínicos e sociais envolvidos na sua gênese. Porém, ainda não se sabe, de forma clara, se esta sindrome pode ser agravada devido à ausência do apoio social. Assim, o objetivo do presente estudo foi analisar a associação entre este e a síndrome da fragilidade em idosos residentes na comunidade. Trata-se de estudo observacional analítico de caráter transversal, com uma amostra de 300 idosos. Foram coletadas informações sobre os dados sociodemográficos e econômicos e de saúde física, fragilidade e apoio social. Para análise estatística, foram realizados o teste qui-quadrado de Pearson, o teste t de student e a regressão logística binária, adotando-se um nível de significância de 5\%. Não foram observadas associações da fragilidade com as variáveis do apoio social, com exceção da modalidade tarefas domésticas ( $p=0,04)$. Na regressão logística, permaneceram associadas a fragilidade à idade (OR $=1,19)$, à atividade física $(O R=2,56)$ e à saúde percebida $(O R=3,33)$. Acredita-se que mais estudos precisam ser desenvolvidos para uma caracterização da vulnerabilidade social, como também os serviços de saúde necessitam reconhecer a importância do apoio social como parte integrante da prestação de cuidados aos idosos.

Palavras-chave Envelhecimento, Idoso fragilizado, Apoio social 


\section{Introdução}

O processo de envelhecimento pode ser compreendido como natural, mediante diminuição progressiva da reserva funcional dos indivíduos, o que em condições normais, não costuma provocar qualquer problema. Porém, em situações de sobrecarga, como no surgimento de doenças, principalmente crônico-degenerativas, associadas a fatores externos como viuvez, aposentadoria irrisória e morte de familiares, pode desencadear uma condição patológica que requeira assistência $^{1}$. A maior ou menor participação destes fatores tem sido associada ao surgimento de incapacidade funcional, dependência em atividades da vida diária $(A V D)$ e, mais recentemente, à síndrome da fragilidade ${ }^{2}$.

Embora não seja sinônimo de tais condições, a síndrome da fragilidade está associada ao maior risco de ocorrência de desfechos clínicos adversos, como declínio funcional, quedas, hospitalização, institucionalização e morte ${ }^{3}$.

Acredita-se que a fisiopatologia da síndrome da fragilidade possa ser agravada devido à ruptura de laços sociais, já que, para alguns autores, a ausência do apoio social afeta os sistemas de defesa do organismo de tal maneira que o indivíduo se torne mais susceptível a doenças ${ }^{4,5}$. De acordo com esse conceito, os laços sociais e o apoio estabelecido por idosos teriam influência na manutenção da saúde, favorecendo condutas adaptativas em situações de estresse ${ }^{6}$.

Apesar dos mecanismos de ação exercidos pelo apoio social no sistema imunológico ainda não terem sido elucidados, duas hipóteses são apresentadas de maneira básica. Na primeira, o apoio social atuaria como "tampão", impedindo a resposta do organismo em forma de doença, em consequência a grandes perdas emocionais ${ }^{7}$. $\mathrm{Na}$ segunda hipótese, poderia reforçar a sensação de controle sobre a própria vida, implicando em efeitos positivos sobre a saúde ${ }^{8}$.

Estudos epidemiológicos já identificaram associação entre o apoio social e a ocorrência de diversos desfechos relacionados à saúde ${ }^{9-11}$. A forte e consistente associação inversa entre maior apoio social e menor mortalidade geral, foi um dos primeiros efeitos identificados sobre a saúde ${ }^{9}$. Investigações posteriores também confirmaram a relação entre a magnitude do apoio social com maior sobrevida após diagnóstico de doença coronariana, acidente vascular cerebral e neoplasias malignas ${ }^{10}$. Além disso, o apoio social também foi associado de maneira inversa com a incidência de insônia, frequência de hipertensão arterial e risco de demência ${ }^{10,11}$.
Diante do exposto, ao considerar que a investigação do apoio social é um elemento importante de um protocolo abrangente na avaliação da saúde dos idosos e pode fazer a diferença entre o sucesso e o fracasso de uma estratégia de intervenção, este estudo objetiva analisar a associação entre o apoio social e a síndrome da fragilidade em idosos residentes na comunidade.

\section{Metodologia}

Esta pesquisa faz parte de um Projeto intitulado "Carga Alostática, fragilidade e funcionalidade em idosos", vinculado à Universidade Federal do Rio Grande do Norte (UFRN) em parceria com a Universitè de Montreal.

Trata-se de um estudo observacional analítico de caráter transversal, realizado no município de Natal (RN), no período de Novembro de 2010 à Julho de 2011. O município possui uma população de 803.739 habitantes. No último Censo Demográfico, a população com 65 anos ou mais representou $7,04 \%$ dos indivíduos, o equivalente a 56.605 idosos.

A população do estudo foi composta por 1.056 idosos residentes no bairro das Rocas, com 65 anos ou mais, e cadastrados na Unidade de Saúde da Família (USF). Apesar de a região estudada ser pobre e pouco desenvolvida, os idosos que ali residiam não se encontravam entre os extremos em níveis de escolaridade, renda e condições sociais. Diante do exposto, e pelo fato de já terem sido realizadas pesquisas anteriores na comunidade, $\mathrm{o}$ que facilitou o acesso à USF e aos idosos, optouse pela realização da pesquisa nesta região.

Para o cálculo do tamanho amostral foram considerados os seguintes parâmetros estatísticos: nível de confiança de 95\%, erros estatísticos máximos de $5,0 \%$ para o tipo I e $20,0 \%$ para o erro de tipo II. Considerando a população do estudo, foi definida uma amostra de 300 indivíduos. Os idosos foram distribuídos de acordo com a proporção estimada de homens e mulheres para cada faixa etária, conforme dados do IBGE.

Os critérios de inclusão foram: possuir 65 anos ou mais, residir na comunidade, ter capacidade de compreensão e comunicação verbal, não apresentar amputação de membro inferior, ser capaz de deambular sozinho, utilizando ou não dispositivo de auxílio à marcha e não possuir limitação severa nas atividades básicas da vida diária (ABVD). Foram excluídos do estudo os idosos que se recusaram a completar todas as fases da entrevista ou que apresentaram escore menor que 17 pontos no Mini-Exame do Estado Mental (MEEM) ${ }^{12}$. 
Para a avaliação dos aspectos biopsicossociais, foi utilizado um questionário estruturado, contendo o conjunto de variáveis descritas a seguir:

- Variáveis sociodemográficas e econômicas: idade; renda pessoal; escolaridade; e hábitos de vida, como consumo de bebida alcoólica, tabagismo e pratica de atividade física nos últimos 3 meses.

- Variáveis de saúde física: presença de comorbidades; percepção geral da saúde, sendo classificada como satisfatória e insatisfatória; e avaliação antropométrica, mediante cálculo do índice de massa corporal (IMC), que corresponde à divisão da massa corporal pelo quadrado da altura, classificado em: baixo peso (IMC $<22 \mathrm{Kg}$ / $\mathrm{m}^{2}$ ); normal $\left(\mathrm{IMC}>22 \mathrm{Kg} / \mathrm{m}^{2}\right.$ e $\left.<27 \mathrm{Kg} / \mathrm{m}^{2}\right)$ e obeso (IMC $\left.>27 \mathrm{Kg} / \mathrm{m}^{2}\right)^{13}$. A massa corporal foi medida por meio de uma balança da marca Filizola $^{\circledR}$, Série 3.134, com divisões de 100g. Para o registro da altura, foi utilizado fita métrica com divisões de $1 \mathrm{~mm}$.

Para avaliação do apoio social, foram utilizados o questionário da pesquisa Saúde, Bemestar e Envelhecimento (SABE) $)^{14,15}$ e o Mapa Mínimo de Relações do Idoso (MMRI) ${ }^{16}$ :

. Questionário da pesquisa SABE: foram avaliados os aspectos estruturais e funcionais do apoio social que incluíam o status de coabitação, indicando se o idoso vivia só ou não; situação conjugal, classificando o idoso como casado ou não casado; índice de frequência de contatos (IFC), que avaliou o grau em que o idoso estava conectado socialmente com outros e correspondeu ao número de contatos mensais com filhos, irmãos, outros familiares e com amigos; índice de diversidade de contatos (IDC), que avaliou a amplitude da rede social e foi construído a partir das mesmas questões do índice anterior, mas calculado como o número de diferentes tipos de contatos ocorridos mais de duas vezes ao mês; índice de frequência de ajudas recebidas (IFAR) e prestadas (IFAP), que correspondeu ao somatório das ajudas mensais efetivamente recebidas ou prestadas por todas as pessoas que faziam parte da rede social do idoso ${ }^{14,15}$.

Os resultados do IFC, IDC, IFAR e IFAP foram divididos em quartis, obtendo os seguintes grupos: IFC (I: < 30 contatos; II: $>30$ e $<60$ contatos; III: $>60$ e $<98$ contatos; e IV: $>98$ contatos); IDC (I: $<2$ contatos; II: $>2$ e $<4$ contatos; III: $>4$ e $<6$ contatos; e IV: $>6$ contatos); IFAR (I: < 61 ajudas; II: > 61 e $<119$ ajudas; III: > 119 e d" 189 ajudas; e IV: $>189$ ajudas); IFAP (I: $<60$ ajudas; II: $>60$ e $<120$ ajudas; III: $>120$ e < 186 ajudas; e IV: $>186$ ajudas).

As variáveis IFC, IDC, IFAR e IFAP foram, posteriormente, dicotomizadas em "baixa frequência” (grupo I) e "outras" (grupo II + grupo III + grupo IV) para melhor visualização dos resultados e condução da análise estatística.

. MMRI: incluiu todas as pessoas que visitam e fazem companhia ao idoso, além daqueles que ajudam financeiramente, nas tarefas domésticas e no cuidado pessoal. Em todas as modalidades de apoio o idoso poderia informar quem efetivamente presta ajuda ou poderia ajudar num momento de dificuldade. O MMRI é formado por 3 círculos, que simbolizam os diversos graus de proximidade: interno, cujos contados são realizados pelo menos uma vez na semana; intermediário, com encontros que ocorrem uma vez por mês; e externo, com contatos ocasionais realizados pelo menos uma vez ao ano. Foi definida como rede de apoio social pequena a presença de até duas pessoas no primeiro círculo; média, entre três e cinco pessoas; e grande, acima de seis pessoas neste círculo ${ }^{16}$.

Para avaliação do fenótipo da fragilidade foram considerados os 5 critérios propostos por Fried et al. ${ }^{3}$ :

. Perda de peso não intencional: identificado mediante a seguinte pergunta: "No último ano, o senhor (a) perdeu mais de 4,5 kg sem intenção (isto é, sem dieta ou exercício)?".

. Fraqueza muscular: avaliada pela diminuição da força de preensão palmar, por meio do dinamômetro $\operatorname{Jamar}^{\circledR}$, mensurada em quilogramas/força (Kgf). Para tal, os participantes do estudo foram orientados a permanecerem sentados, estando o ombro aduzido, cotovelo fletido à $90^{\circ}$, o punho entre 0 a $30^{\circ}$ de extensão. Foi dado um comando verbal pelo examinador, em volume alto, no momento em que o idoso deveria puxar a alça do dinamômetro e mantê-la por seis segundos. Três medidas foram realizadas na mão dominante do voluntário e considerou-se a medida de maior valor. Foram adotados, como pontos de corte (PC) para fragilidade, os menores quintis estratificados por gênero e quartis do IMC: (homens: $0 \mathrm{Kg} / \mathrm{m}^{2}<\mathrm{IMC}<23,59 \mathrm{Kg} / \mathrm{m}^{2}$ à $\mathrm{PC}<23 \mathrm{Kgf} ; 23,60 \mathrm{Kg} / \mathrm{m}^{2}<\mathrm{IMC}<25,90 \mathrm{Kg} / \mathrm{m}^{2}$ à PC $<23,20 \mathrm{Kgf} ; 25,91 \mathrm{Kg} / \mathrm{m}^{2}<\mathrm{IMC}<28,76 \mathrm{Kg} /$ $\mathrm{m}^{2}$ à $\mathrm{PC}<25 \mathrm{Kgf} ; \mathrm{IMC}>28,77 \mathrm{Kg} / \mathrm{m}^{2}$ à $\mathrm{PC}<$ 28Kgf; mulheres: $0 \mathrm{Kg} / \mathrm{m}^{2}<\mathrm{IMC}<23,55 \mathrm{Kg} / \mathrm{m}^{2}$ à $\mathrm{PC}<12 \mathrm{Kgf} ; 23,56 \mathrm{Kg} / \mathrm{m}^{2}<\mathrm{IMC}<27,25 \mathrm{Kg} / \mathrm{m}^{2}$ à $\mathrm{PC}<14 \mathrm{Kgf} ; 27,26 \mathrm{Kg} / \mathrm{m}^{2}<\mathrm{IMC}<30,50 \mathrm{Kg} / \mathrm{m}^{2}$ à $\mathrm{PC}<15,40 \mathrm{Kgf} ; \mathrm{IMC}>30,51 \mathrm{Kg} / \mathrm{m}^{2}$ à $\left.>14 \mathrm{Kgf}\right)$.

. Nível de atividade física: foi avaliado por intermédio da versão curta do Questionário Internacional de Atividade Física (IPAQ ${ }^{17}$. Esta versão consta de informações que permitem estimar o tempo despendido na última semana, com duração mínima de 10 minutos contínuos, em 
três diferentes atividades: caminhada, de moderada intensidade e de intensidade vigorosa. Para o cálculo dos escores de atividade física foram empregados os procedimentos descritos nas $\mathrm{Di}$ retrizes para Processamento e Análise de Dados do IPAQ, promovendo os resultados em METminutos/semana. Para cada atividade física foi feita a multiplicação do tempo semanal de prática (em minutos) por uma constante referente à intensidade das atividades realizadas, tendo como base o equivalente metabólico (MET) em repouso. Foi utilizada a constante 3,3 MET para caminhada, 4,0 MET para atividades de intensidade moderada e 8,0 MET para atividades de intensidade vigorosa. O cálculo do escore de atividade física total foi obtido pela soma dos gastos energéticos de cada atividade. Os resultados calculados em MET-minutos/semana foram multiplicados pelo peso do idoso e dividido por 60 quilogramas. Os menores quintis destes resultados, estratificados por gênero, foram identificados e utilizados como ponto de corte para classificar o baixo nível de atividade física. O ponto de corte para homens e mulheres foi de $0,0 \mathrm{Kcal} /$ semana.

- Exaustão: foi avaliada pelo autorrelato de fadiga de acordo com as questões 7 ("Sinto que tudo que fiz foi muito custoso") e 20 ("Eu me sinto desanimado") da escala de depressão do Center for Epidemiological Studies (CES-D) ${ }^{3}$. As respostas a cada uma das questões foram dadas segundo a frequência com que cada sintoma esteve precedente na última semana à aplicação do instrumento, podendo ser: raramente ou nunca (menos que 1 dia); poucas vezes (1-2 dias); às vezes (3-4 dias); quase sempre ou sempre (5-7 dias). Os idosos que responderam "às vezes" ou "quase sempre ou sempre" em qualquer uma das duas questões preencheram o critério de fragilidade para este item.

. Lentidão: foi calculada pelo tempo de marcha (em segundos) gasto para percorrer uma distância de 4,6 metros, sendo solicitado que o idoso deambulasse com a sua velocidade de marcha atual e que realizasse o teste com seu calçado habitual. Caso fosse necessário, poderia utilizar algum dispositivo de auxílio à marcha. Os resultados para este item, transformados na unidade metro/segundo $(\mathrm{m} / \mathrm{s})$, foram ajustados pela mediana da altura para homens e para mulheres, sendo os menores quintis, utilizados como ponto de corte: (homens: $0 \mathrm{~m}<$ altura $<1,64 \mathrm{~m}$ à $\mathrm{PC}<0,60 \mathrm{~m} / \mathrm{s}$; altura $>1,64 \mathrm{~m}$ à $\mathrm{PC}<0,67 \mathrm{~m} / \mathrm{s}$; mulheres: $0 \mathrm{~m}<$ altura $<1,50 \mathrm{~m}$ à $\mathrm{PC}<0,51 \mathrm{~m} / \mathrm{s}$; altura $>1,50 \mathrm{~m}$ à $\mathrm{PC}<0,63 \mathrm{~m} / \mathrm{s}$ ).

Dessa forma, os idosos receberam um ponto para cada critério positivo, resultando um esco- re de fragilidade que variou de 0 a 5 . Os idosos que apresentaram escores de 3 a 5 foram classificados como frágeis, 1 ou 2 caracterizavam indivíduos pré-frágeis e escore zero, idosos não frágeis. Posteriormente, a variável fragilidade foi dicotomizada em frágil e não frágil (pré-frágil + não frágil), no intuito de melhor ajuste desta para a análise estatística.

Todas as entrevistas foram realizadas nas residências dos idosos, por pesquisadores do curso de Fisioterapia da UFRN, que foram devidamente treinados para a utilização do instrumento.

Os dados obtidos foram tabulados, armazenados e analisados no software Statistical Package for the Social Science (SPSS) versão 17.0. Os mesmos foram submetidos à análise estatística descritiva e analítica, adotando-se um nível de significância de 5\%. Na abordagem descritiva, as variáveis categóricas foram descritas mediante frequências absolutas e relativas e as variáveis quantitativas, por meio das médias, com desvio padrão $( \pm \mathrm{dp})$.

$\mathrm{Na}$ abordagem analítica, foi realizada análise bivariada por meio do teste qui-quadrado de Pearson $\left(\chi^{2}\right)$ para se observarem as possíveis associações existentes entre as variáveis independentes categóricas e as dependentes. Já o teste t de student foi utilizado para comparação de médias de variáveis quantitativas. Em seguida foi realizada análise multivariada, por regressão logística binária. Todas as variáveis independentes foram incluídas no modelo inicial e então foram regressivamente excluídas até que somente aquelas com valor $\mathrm{p}<0,05$ e intervalo de confiança (IC) de 95\% foram mantidas, com cálculo dos odds ratios (OR) ajustados.

Este estudo foi submetido à apreciação do Comitê de Ética em Pesquisa do Hospital Universitário Onofre Lopes (HUOL) da UFRN e aprovado, de acordo com as atribuições da Resolução no 196/96 ${ }^{18}$ do Conselho Nacional de Saúde.

\section{Resultados}

A amostra foi composta por 300 indivíduos, com faixa etária prevalente entre 65 a 69 anos $(29,0 \%)$ e média de 74,3 anos $( \pm 6,9)$. Observou-se predomínio de idosos do sexo feminino, com renda pessoal de 1 a 3 salários mínimos e baixo nível de escolaridade, com média de 4,7 anos de estudo $( \pm 3,8)$. As demais características sociodemográficas são apresentadas na Tabela 1.

Em relação aos aspectos relacionados à saúde (Tabela 2), a maioria dos idosos considerou 
Tabela 1. Distribuição das variáveis

sociodemográficas e econômicas entre os idosos,

Natal (RN), 2011.

\begin{tabular}{|c|c|c|}
\hline Variáveis & $\mathbf{n}=\mathbf{3 0 0}$ & $\%$ \\
\hline \multicolumn{3}{|l|}{ Sexo } \\
\hline Feminino & 202 & 67,3 \\
\hline Masculino & 98 & 32,7 \\
\hline \multicolumn{3}{|l|}{ Faixa etária } \\
\hline 65-69 anos & 87 & 29,0 \\
\hline 70-74 anos & 80 & 26,7 \\
\hline 75-79 anos & 59 & 19,7 \\
\hline$>80$ anos & 74 & 24,7 \\
\hline \multicolumn{3}{|l|}{ Renda pessoal } \\
\hline Nenhuma & 9 & 3,0 \\
\hline < 1 salário mínimo & 9 & 3,0 \\
\hline 1 - 3 salários mínimos & 245 & 81,7 \\
\hline 4 - 5 salários mínimos & 26 & 8,7 \\
\hline > 5 salários mínimos & 11 & 3,7 \\
\hline \multicolumn{3}{|l|}{ Escolaridade } \\
\hline$<1$ ano & 45 & 15,0 \\
\hline $1-4$ anos & 108 & 36,0 \\
\hline $5-8$ anos & 105 & 35,0 \\
\hline $9-12$ anos & 29 & 9,7 \\
\hline$>12$ anos & 8 & 2,7 \\
\hline $\mathrm{NS} / \mathrm{NR}$ & 5 & 1,6 \\
\hline \multicolumn{3}{|c|}{ Consumo de bebida alcoólica } \\
\hline Sim & 104 & 34,7 \\
\hline Não & 195 & 65,0 \\
\hline $\mathrm{NS} / \mathrm{NR}$ & 1 & 0,3 \\
\hline \multicolumn{3}{|l|}{ Tabagismo } \\
\hline Sim & 122 & 40,7 \\
\hline Não & 178 & 59,3 \\
\hline \multicolumn{3}{|l|}{ Atividade Física } \\
\hline Sim & 96 & 32,0 \\
\hline Não & 204 & 68,0 \\
\hline
\end{tabular}

NS/NR = não sabe ou não respondeu.

seu estado de saúde como insatisfatório. Verificou-se maior proporção de participantes com IMC acima de $27 \mathrm{~kg} / \mathrm{m}^{2}$, com média de $27,0 \mathrm{~kg} /$ $\mathrm{m}^{2}( \pm 5,2)$. Além disto, constatou-se que os entrevistados obtiveram escore médio no MEEM de 22,2 pontos $( \pm 3,6)$. Dentre as comorbidades mais prevalentes, destacaram-se a HAS, o reumatismo e a diabetes mellitus.

Com base no fenótipo proposto para detectar a fragilidade, $18,3 \%$ da amostra foi categorizada como frágil, conforme a Tabela 2. Dentre os critérios de fragilidade mais frequentemente encontrados, destacaram-se a exaustão $(38,7 \%)$ e a perda de peso não intencional $(30,7 \%)$, seguidos do baixo nível de atividade física $(26,7 \%)$, fraqueza $(22,3 \%)$ e lentidão $(19,0 \%)$.
Tabela 2. Distribuição das variáveis de saúde física entre os idosos, Natal (RN), 2011.

\begin{tabular}{|c|c|c|}
\hline Variáveis & $\mathrm{n}=\mathbf{3 0 0}$ & $\%$ \\
\hline \multicolumn{3}{|l|}{ Percepção da saúde } \\
\hline Satisfatória & 81 & 27,0 \\
\hline Insatisfatória & 219 & 73,0 \\
\hline
\end{tabular}

14,3

$22 \mathrm{~kg} / \mathrm{m}^{2}$

$22-27 \mathrm{~kg} / \mathrm{m}^{2}$

$>27 \mathrm{~kg} / \mathrm{m}^{2}$

$111 \quad 37,0$

14648,7

Hipertensão Arterial Sistêmica

Sim

Não

$203 \quad 67,7$

$97 \quad 32,3$

Diabetes mellitus

Sim

Não

NS/NR

$89 \quad 29,7$

20769,0

$4 \quad 1,3$

Acidente Vascular Cerebral

Sim

Não

NS/NR

$27 \quad 9,09$

$272 \quad 0,7$

Câncer

Sim

Não

NS/NR

Doença pulmonar crônica

Sim

Não

Doença cardíaca

Sim

Não

NS/NR

Reumatismo

Sim

Não

NS/NR

Depressão

Sim

Não

NS/NR

Fragilidade

Não Frágil

Pré-frágil

Frágil

NA

$\mathrm{NS} / \mathrm{NR}$ = não sabe ou não respondeu; $\mathrm{NA}=$ não se aplica.

$\mathrm{Na}$ Tabela 3 são apresentados os resultados referentes aos aspectos estruturais das redes de apoio dos entrevistados. Observou-se que a grande maioria dos idosos não morava só e foram classificados como não casados (solteiros, viúvos, separados/divorciados). Em relação ao IFC 
Tabela 3. Distribuição das variáveis do apoio social e MMRI entre os idosos, Natal (RN), 2011.

\begin{tabular}{|c|c|c|}
\hline Variáveis & $\mathbf{n}=\mathbf{3 0 0}$ & $\%$ \\
\hline \multicolumn{3}{|l|}{ Mora só } \\
\hline $\operatorname{Sim}$ & 23 & 7,7 \\
\hline Não & 277 & 92,3 \\
\hline \multicolumn{3}{|l|}{ Situação conjugal } \\
\hline Casados & 131 & 43,7 \\
\hline Não casados & 169 & 56,3 \\
\hline \multicolumn{3}{|c|}{ Índice de frequência de contatos } \\
\hline Baixa frequência & 86 & 28,7 \\
\hline Outras & 214 & 71,3 \\
\hline \multicolumn{3}{|c|}{ Índice de diversidade de contato } \\
\hline Baixa frequência & 101 & 33,7 \\
\hline Outras & 199 & 66,3 \\
\hline \multicolumn{3}{|c|}{$\begin{array}{l}\text { Índice de frequência de ajudas } \\
\text { recebidas }\end{array}$} \\
\hline Baixa frequência & 79 & 26,3 \\
\hline Outras & 221 & 73,7 \\
\hline \multicolumn{3}{|c|}{$\begin{array}{l}\text { Índice de frequência de ajudas } \\
\text { prestadas }\end{array}$} \\
\hline Baixa frequência & 77 & 25,7 \\
\hline Outras & 223 & 74,3 \\
\hline \multicolumn{3}{|l|}{ Visita } \\
\hline Pequena & 144 & 48,0 \\
\hline Média & 104 & 34,7 \\
\hline Grande & 52 & 17,3 \\
\hline \multicolumn{3}{|l|}{ Companhia } \\
\hline Pequena & 155 & 51,7 \\
\hline Média & 103 & 34,3 \\
\hline Grande & 42 & 14,0 \\
\hline \multicolumn{3}{|l|}{ Tarefas domésticas } \\
\hline Pequena & 247 & 82,3 \\
\hline Média & 50 & 16,7 \\
\hline Grande & 3 & 1,0 \\
\hline \multicolumn{3}{|l|}{ Cuidado pessoal } \\
\hline Pequena & 204 & 68,0 \\
\hline Média & 75 & 25,0 \\
\hline Grande & 21 & 7,0 \\
\hline \multicolumn{3}{|l|}{ Ajuda financeira } \\
\hline Pequena & 257 & 85,7 \\
\hline Média & 29 & 9,6 \\
\hline Grande & 14 & 4,7 \\
\hline
\end{tabular}

e IDC, $28,7 \%$ dos idosos apresentaram baixa frequência de contatos e $33,7 \%$ foram considerados com baixa diversidade de contatos.

Quanto aos aspectos funcionais do apoio social, a Tabela 3 mostra que os dados referentes ao IFAR foram semelhantes ao do IFAP, em que $26,3 \%$ e $25,7 \%$ dos idosos apresentaram baixa frequência de ajudas recebidas e prestadas, respectivamente.
Em relação ao MMRI, constatou-se que o tamanho da rede social foi considerado pequeno para as diversas modalidades de apoio: visita $(48,0 \%)$; companhia $(51,7 \%)$; tarefas domésticas $(82,3 \%)$; cuidado pessoal $(68,0 \%)$; e ajuda financeira $(85,7 \%)$. Os resultados podem ser observados na Tabela 3.

$\mathrm{Na}$ análise bivariada, algumas variáveis foram recategorizadas, com o objetivo de melhor ajustá-las para visualização dos resultados. Para tal, foram consideradas as suas distribuições ou modelos similares na literatura, conforme descrições apresentadas na metodologia. Nesta etapa, 5 idosos foram excluídos por possuírem dados incompletos acerca das variáveis relativas à fragilidade, obtendo-se uma amostra total de 295 casos válidos. Dentre as variáveis referentes ao apoio social, observou-se que não houve associação significativa entre as variáveis relativas aos aspectos estruturais e funcionais do apoio social e a fragilidade em idosos. Referente às variáveis relacionadas com as modalidade de apoio do MMRI, apenas a ajuda nas tarefas domésticas foi associada significativamente à fragilidade ( $\mathrm{p}$ $=0,04$ ), como pode ser observado na Tabela 4 .

$\mathrm{Na}$ análise multivariada, a partir de uma regressão logística binária, observou-se que nenhuma das variáveis relacionadas ao apoio social permaneceu no modelo final de regressão logística (Tabela 5).

\section{Discussão}

Este estudo teve como objetivo investigar a associação entre o apoio social e a síndrome da fragilidade por meio de um desenho transversal em uma amostra de idosos residentes na comunidade.

Em relação ao status de coabitação, a proporção de idosos que viviam sozinhos $(7,7 \%)$ foi semelhante à encontrada em estudo realizado no Piauí $(7,6 \%)^{19}$. No entanto, esta proporção foi inferior à encontrada em outros estudos epidemiológicos, realizados em outras regiões do país ${ }^{19,20}$. Pagotto et al..$^{20}$ e Duarte et al. ${ }^{21}$ constataram que $9,4 \%$ e $13,1 \%$ dos idosos moravam só. Segundo Cesar et al. ${ }^{19}$, os principais determinantes de viver sozinho são a renda familiar e pessoal, como também a satisfação com os rendimentos. Como as diferenças socioeconômicas ainda existem entre as regiões do país, este pode ser um fator determinante para os resultados encontrados.

Concernente à situação conjugal, observouse que $43,7 \%$ dos idosos eram casados ou possuíam união estável (56,3\%). Estudos anterio- 
Tabela 4. Distribuição da fragilidade, de acordo com as variáveis de apoio social e MMRI dos idosos, Natal (RN), 2011.

\begin{tabular}{|c|c|c|c|}
\hline Variáveis & Não frágil ${ }^{*}$ & Frágil $^{*}$ & $\mathbf{p}$ \\
\hline Mora sozinho $(n, \%)$ & & & 0,53 \\
\hline Sim & $19(86,4 \%)$ & $3(13,6 \%)$ & \\
\hline Não & $221(81,0 \%)$ & $52(19,0 \%)$ & \\
\hline Situação conjugal (n, \%) & & & 0,46 \\
\hline Casados & $131(79,9 \%)$ & $33(20,1 \%)$ & \\
\hline Não-casados & $109(83,2 \%)$ & $22(16,8 \%)$ & \\
\hline $\operatorname{IFC}(\mathrm{n}, \%)$ & & & 0,91 \\
\hline Baixa frequência & $68(81,0 \%)$ & $16(19,0 \%)$ & \\
\hline Outras & $172(81,5 \%)$ & $39(18,5 \%)$ & \\
\hline IDC (n, \%) & & & 0,29 \\
\hline Baixa frequência & $83(84,7 \%)$ & $15(15,3 \%)$ & \\
\hline Outras & $157(79,7 \%)$ & $40(20,3 \%)$ & \\
\hline $\operatorname{IFAR}(n, \%)$ & & & 0,13 \\
\hline Baixa frequência & $67(87,0 \%)$ & $10(13,0 \%)$ & \\
\hline Outras & $173(79,4 \%)$ & $45(20,6 \%)$ & \\
\hline IFAP $(n, \%)$ & & & 0,99 \\
\hline Baixa frequência & $61(81,3 \%)$ & $14(18.7 \%)$ & \\
\hline Outras & $179(81,4 \%)$ & $41(18,6 \%)$ & \\
\hline Visita (n, \%) & & & 0,88 \\
\hline Pequena & $116(81,7 \%)$ & $26(18,3 \%)$ & \\
\hline Outras & $124(81,0 \%)$ & $29(19,0 \%)$ & \\
\hline Companhia (n, \%) & & & 0,68 \\
\hline Pequena & $125(82,2 \%)$ & $27(17,8 \%)$ & \\
\hline Outras & $115(80,4 \%)$ & $28(19,6 \%)$ & \\
\hline Tarefas domésticas (n, \%) & & & 0,04 \\
\hline Pequena & $202(83,5 \%)$ & $40(16,5 \%)$ & \\
\hline Outras & $38(71,7 \%)$ & $15(28,3 \%)$ & \\
\hline Cuidado pessoal (n, \%) & & & 0,87 \\
\hline Pequena & $164(81,6 \%)$ & $37(18,4 \%)$ & \\
\hline Outras & $76(80,9 \%)$ & $18(19,1 \%)$ & \\
\hline Ajuda financeira $(n, \%)$ & & & 0,66 \\
\hline Pequena & $204(81,0 \%)$ & $48(19,0 \%)$ & \\
\hline Outras & $36(83,7 \%)$ & $7(16,3 \%)$ & \\
\hline
\end{tabular}

Tabela 5. Modelo final de regressão logística binária para variáveis associadas a síndrome da fragilidade, Natal (RN), 2011.

\begin{tabular}{lccc}
\hline \multicolumn{1}{c}{ Variáveis } & $\mathbf{p}$ & OR $_{\text {ajustada }}$ & IC 95\% \\
\hline $\begin{array}{l}\text { Idade (em anos) } \\
\text { Atividade física }\end{array}$ & $<0,001$ & 1,19 & $1,06-1,17$ \\
$\quad$ Sim & 0,002 & 2,56 & $1,10-5,98$ \\
$\quad$ Não & & & \\
Saúde percebida \\
$\quad \begin{array}{l}\text { Satisfatória } \\
\text { Insatisfatória }\end{array}$ & 0,001 & 3,33 & $1,23-8,97$ \\
\hline
\end{tabular}

res $^{22,23}$ obtiveram resultados mais expressivos ao constatarem que $57,0 \%$ e $52,5 \%$ da população idosa possuía companheiro. Para Camarano ${ }^{24}$, após separação do cônjuge ou viuvez, as idosas têm tendência a permanecerem sozinhas. Já os homens se casam novamente. Tal fato talvez justifique a maior prevalência de idosos "não casados" encontrada, visto que a amostra foi composta principalmente de mulheres.

Constatou-se que 28,7\% dos idosos possuíam baixa frequência de contatos (IFC), referente a menos de 30 contatos mensais. Além disso, observou-se que $33,7 \%$ do total de idosos possuíam 2 ou menos contatos regulares (baixo IDC). Estudo com idosos franceses constatou que 38,3\% mulheres e $41,0 \%$ homens possuíam até 2 contatos regulares ${ }^{25}$. Já Cornwell et al. ${ }^{26}$ encontrou que $27,7 \%$ dos idosos possuíam baixa frequência de pessoas para discutir "assuntos importantes".

Apenas 26,3\% dos idosos obtiveram baixa frequência de ajudas recebidas ( $<61$ ajudas men- 
sais). Observou-se também que as ajudas nas tarefas domésticas (80,3\%) e companhia $(77,7 \%)$ foram as mais prevalentes. Lebrão e Duarte et al. ${ }^{21}$ observaram que $78,0 \%$ dos idosos recebiam ajudas regulares em serviços e $20,0 \%$ por intermédio de companhia. Em outro estudo, Avlund et al. ${ }^{27}$ observaram que apenas $25 \%$ das mulheres e $7 \%$ dos homens receberam ajuda nas tarefas domésticas no mês anterior à entrevista.

Já em relação à ajuda oferecida pelos idosos, observou-se pequena prevalência $(25,7 \%)$ de idosos classificados com baixa frequência $(<60$ ajudas mensais). Guedea et al. ${ }^{28}$ afirmam que ser provedor de apoio contribui significativamente para o aumento da satisfação do idoso com a vida e para a diminuição dos seus afetos negativos.

Due et al. ${ }^{29}$ observaram que, geralmente, as mulheres possuem redes de apoio maiores e mais próximas. Esta afirmação pode justificar a maior média de contatos e ajudas obtidas no atual estudo. No entanto, as mulheres nem sempre têm os mesmo benefícios de saúde de suas redes sociais quanto os homens, visto que os tipos de contatos e a ajuda oferecida por estes diferem entre os gêneros. Enquanto as mulheres possuem mais contatos com seus filhos e pouco contato com amigos, os homens geralmente possuem a mesma quantidade de contato com ambos.

Alvarenga et al..$^{30}$ observaram que $30,6 \%$ dos entrevistados possuíam rede pequena para a modalidade visita; $61,1 \%$ para companhia; $78,3 \%$ para tarefas domésticas; $71,4 \%$ para cuidado pessoal; e $54,5 \%$ para ajuda financeira. Esses resultados são semelhantes ao do presente estudo nas modalidades tarefas domésticas e cuidado pessoal.

Quanto à presença de fragilidade, observouse maior proporção de idosos pré-frágeis $(55,3 \%)$, seguido dos não frágeis $(26,1 \%)$ e frágeis $(18,6 \%)$. Os resultados de Freiheit et al. ${ }^{31}$ corroboram com os achados do presente estudo, ao constatarem que $55,2 \%$ da população idosa canadense eram pré-frágeis, 25,6\% não frágeis e 19,2\% frágeis. Santos-Eggimann et al..$^{32}$ também encontraram maiores proporções de idosos europeus pré-frágeis $(42,3 \%)$ e não frágeis $(40,7 \%)$, com menor porcentagem de frágeis (17.0\%). Espinoza et al. ${ }^{33}$ também reforçam estes achados ao afirmarem que as prevalências de idosos americanos préfrágeis, não frágeis e frágeis foram, respectivamente, $53,1 \%, 37,1 \%$ e $9,8 \%$. No entanto, dados do estudo $\mathrm{SABE}^{34}$ mostraram que $40,0 \%$ dos idosos brasileiros foram considerados frágeis. Esta maior proporção de frágeis encontrada no estudo SABE justifica-se pela utilização de outros instrumentos para categorização da fragilidade.
Considerando os critérios de rastreamento da fragilidade, observou-se que as frequências de fraqueza e lentidão corroboram com dados de estudos prévios ${ }^{35,36}$. No entanto, a exaustão, o baixo nível de atividade física e a perda de peso não intencional, que foram os critérios mais frequentes, possuem proporções inferiores em diversos estu$\operatorname{dos}^{37,38}$. A elevada queixa de fadiga pode ser justificada pelo fato desta ser mais prevalente entre as mulheres, provavelmente como resultado da realização de tarefas domésticas, muitas vezes sob condições de baixa força muscular ${ }^{37}$. Isto pode ser decorrente das diferenças entre as populações estudadas, como idade e condições de saúde ${ }^{39}$. Em relação ao baixo nível de atividade física, devese considerar que o questionário de atividades de lazer de Minnesota, proposto por Fried et al. ${ }^{3}$, não tem sua validação assegurada em todas as populações. Sendo assim, estudiosos têm utilizado outros protocolos ${ }^{35,36}$ para a avaliação deste critério, o que dificulta sua comparação de forma fidedigna. No presente estudo foi utilizado o IPAQ, o que não comprometeu a validade interna do mesmo, visto que este instrumento já foi empregado como critério de fragilidade pela pesquisa $\mathrm{SABE}^{40}$, referência em estudos epidemiológicos com idosos no Brasil. Quanto ao critério perda de peso, a maior prevalência no nosso estudo pode estar relacionada com as diferenças entre as populações estudadas como, por exemplo, na altura e peso, o que repercute de forma singular para cada população.

Assim como em estudos anteriores, a fragilidade foi associada à presença de patologias ${ }^{36,38,41}$, especialmente HAS, Diabetes mellitus, AVC e doença cardíaca. Além disso, dados do Cardiovascular Health Study ${ }^{42}$ sugerem que a fragilidade pode contribuir para o desenvolvimento ou a progressão de doenças crônicas, possivelmente por intermédio dos níveis mais baixos de atividade, ou mediante outros caminhos que afetam algum mecanismo biológico básico essencial para a manutenção da homeostase, como a inflamação o equilíbrio simpático-parassimpático.

A associação da função cognitiva com a fragilidade corrobora com achados de outros estu$\operatorname{dos}^{37,41}$. Sendo assim, autores sugerem que a função cognitiva seja incluída nos critérios usados para definir fragilidade. Estes afirmam que a adição deste critério resulta em uma melhor identificação de idosos com vulnerabilidade intrínseca, enquanto apenas os domínios de função física são considerados ${ }^{37,41}$.

Dentre as variáveis sociodemográficas, econômicas e de saúde física, apenas a idade, o se- 
dentarismo e a saúde percebida insatisfatória foram as que permaneceram no modelo de regressão logística, com forte associação para a presença de fragilidade.

Observou-se que vários estudos também encontraram associações entre a fragilidade e a ida$\mathrm{de}^{34,37,41}$. Considera-se que sua associação com a idade avançada tem explicação pela própria característica do processo de envelhecimento, uma vez que todos os sistemas do corpo sofrem perdas tanto nos aspectos estruturais como funcionais ${ }^{1}$. Quanto à atividade física, Costa e $\mathrm{Neri}^{37}$ observaram que idosos sedentários foram associados significativamente aos indicadores de fragilidade referentes à baixa força de preensão e lentidão de marcha. Além disto, também já está bem estabelecido na literatura que a atividade física ajuda na manutenção da saúde e funcionalidade em idosos ${ }^{35}$.

Neste sentido, destaca-se a importância da adoção da prática regular de exercícios físicos, porém sabe-se que esta pode ser questão de oportunidade de acesso, de valores culturais associados a papéis de gênero, de opção, de motivação e de crenças acerca de seus benefícios, estas associadas à educação ${ }^{37}$.

Já em relação à percepção da saúde, ÁvilaFunes et al. ${ }^{41}$ também constataram associação significativa desta variável com a fragilidade. Estudiosos afirmaram que a auto-avaliação insatisfatória do estado de saúde apresentou associações significativas com presença de doenças ${ }^{20,42.43}$, o que pode justificar sua forte relação com a presença de fragilidade em idosos.

No presente estudo, não foram observadas associações da fragilidade com as variáveis do apoio social, com exceção da modalidade tarefas domésticas do MMRI. Corroborando com os nossos achados, Andrew et al. ${ }^{44}$, por intermédio de estudo longitudinal do Canadian Study of Healthe Aging National Population Health Survey, observaram fraca correlação entre a vulnerabilidade social e a fragilidade. Algumas explicações desses achados são sugeridas. Para Cohen e Wi$11 s^{45}$, o efeito tampão do apoio social raramente ou nunca é demonstrado em situações onde não exista um fator estressante específico a ser tamponado. Portanto, o apoio social pode não ter sido associado à fragilidade por esta ser considerada como um declínio cumulativo nas reservas fisiológicas ${ }^{3}$, porém ainda inexistindo o fator estressante.

Além disto, observou-se que os idosos do presente estudo estão bem conectados socialmente, com uma maior variedade de contatos e ajuda oferecida por estes. Somado a estes fatores, a opção de viver só e possuir estado civil "não casado", considerados fatores de vulnerabilidade social, podem ser reflexos de preferências pessoais e sensação de bem-estar, visto que muitos idosos também recebiam visitas e possuíam companhia de familiares, amigos e pessoas da comunidade. Já a elevada proporção de idosos com baixa frequência de ajuda financeira no presente estudo justifica-se pelo fato da maioria desses possuírem renda.

Observou-se que não houve relação entre a fragilidade e a ajuda nos cuidados pessoais. Este fato pode estar relacionado com a seleção da amostra, visto que idosos com limitações severas nas ABVD não foram incluídos no estudo. Dessa forma, como nossa amostra constava de idosos menos comprometidos funcionalmente, justifica-se a associação do apoio social com a ajuda em tarefas domésticas, que é um tipo de atividade instrumental da vida diária (AIVD). Duarte et al. ${ }^{21}$ corroboram com esta afirmação ao reforçar que a relação hierárquica de perdas de habilidades funcionais que ocorrem no envelhecimento se direcionam das AIVD para as ABVD.

Por fim, nossos resultados, assim como os de Andrew et al. ${ }^{44}$, talvez se justifiquem pela operacionalização do apoio social basear-se em autorrelatos de dados ao invés de terem sido objetivamente definidos. Assim, é possível que alguns indivíduos tenham superestimado ou subestimado suas frequências e diversidades de contatos e o apoio estabelecido por estas. Além disto, não foi avaliada a qualidade do apoio social fornecido, como também se as trocas sociais foram positivas ou negativas para os idosos. Portanto, são necessários estudos mais aprofundados das distinções entre os aspectos objetivos e subjetivos do apoio social.

A representatividade da amostra e o baixo percentual de recusas foram pontos positivos do presente estudo. Entre as limitações, o desenho transversal limitou a possibilidade de estudar a temporalidade entre a fragilidade e o apoio social.

Por fim, o presente estudo investigou a associação entre a síndrome da fragilidade e o apoio social em uma população de idosos comunitários, e mostrou que, dentre as variáveis relacionadas com o apoio social, apenas a ajuda nas tarefas domésticas esteve associada significativamente à fragilidade. No entanto, mais pesquisas precisam ser desenvolvidas para caracterização da vulnerabilidade social, como também serviços de saúde e representantes governamentais necessitam reconhecer a importância do apoio social como parte integrante da prestação de cuidados aos idosos. 


\section{Colaboradores}

FLJS Amaral, ACC Maciel, AFF Nascimento e RO Guerra contribuíram na concepção e planejamento; pesquisa e metodologia; análise e interpretação dos dados; elaboração do manuscrito e revisão crítica do conteúdo; e aprovação da versão final do mesmo.

\section{Referências}

1. Filho ET, Papaléo Neto M. Geriatria: Fundamentos, Clínica e Terapêutica. $2^{a}$ Edição. São Paulo: Ed. Atheneu; 2006.

2. Strawbridge WJ, Shelma SJ, Balfour JL, Higby HR, Kaplan GA. Antecedents of frailty over three decades in an older cohort. J Gerontol B Psychol Sci Soc Sci 1998; 53(1):9-16.

3. Fried LP, Tangen CM, Walston J, Newman AB, Hirsch C, Gottdiener J, Seeman T, Tracy R, Kop WJ, Burke G, McBurnie MA. Frailty in older adults: evidence for a phenotype. J Gerontol A Biol Sci Med Sci 2001; 56(3):M146-M156.

4. Morley J, Perry HM, Miller DK. Something about frailty. J Gerontol A Biol Sci Med Sci 2002; 57A(11) M698-M704.

5. Woo J, Goggins W, Sham A. Social determinants of frailty. Gerontology 2005; 51(6):402-408.

6. Griep RH, Chor D, Faerstein E, Werneck GL, Lopes CS. Validade de constructo de escala de apoio social do Medical Outcomes Study adaptada para o português no Estudo Pró-Saúde. Cad Saude Publica 2005; 21(3):703-714.

7. Cohen S, Wills TA. Stress, social support, and the buffering hypothesis. Psychological Bulletin 1985; 98(2):310-357.

8. Rodin J. Aging and health: effects of the sense of control. Science 1986; 233(4770):1271-1276.

9. Kaplan GA, Salonen JT, Cohen RD, Brand RJ, Syme SL, Puska P. Social connections and mortality from all causes and from cardiovascular disease: Pro spective evidence from Eastern Finland. Am J Epidemiol 1988; 128(2):370-380.

10. Vogt TM, Mullooly JP, Ernst D, Pope CR, Hollis JF Social Networks as predictors of ischemic heart disease, cancer, stroke and hypertension: Incidence, survival and mortality. J Clin Epidemiol 1992; 45(6): 659-666.

11. Dressler WW, Balieiro MC, Santos JE. The cultural construction of social support in Brazil: Associations with health outcomes. Cult Med Psychiatr 1997; 2(3):303-335.

12. Laks J, Batista EMR, Guilherme ERL, Contino AL, Faria ME, Figueira I, Engelhardt E. O mini exame do estado mental em idosos de uma comunidade: dados parciais de Santo Antonio de Pádua, Rio de Janeiro. Arq Neuropsiquiatr 2003; 61(3B):782-785.

13. Cervi A, Franceschini SCC, Priore SE. Análise crítica do uso do índice de massa corporal para idosos. Rev Nutr 2005; 18(6):765-775.

14. Lund R, Modvig J, Due P, Holstein BE. Stability and change in structural social relations as preditor of mortality among elderly women and men. Eur Epidemiol 2000; 16(2):1087-1097.

15. Rosa TEC, Benício MHD, Alves MCGP, Lebrão ML. Aspectos estruturais e funcionais do apoio social de idosos do Município de São Paulo, Brasil. Cad Saude Publica 2007; 23(12):2982-2992.

16. Domingues MARC. Mapa Mínimo de Relações: adaptação de um instrumento gráfico para configuraçäo da rede de suporte social do idoso [tese]. São Paulo (SP): Universidade de São Paulo; 2004. 
17. International Physical Activity Questionnaire (IPAQ). Guidelines for Data Processing and Analysis of the International Physical Activity Questionnaire (IPAQ) - Short and Long Forms. 2005 [acessado 2013 maio 8]. Disponível em: http://www.ipaq.ki.se/ scoring.pdf

18. Brasil. Ministério da Saúde (MS). Conselho Nacional de Saúde. Resolução no 196 de 10 de outubro de 1996. Diretrizes e Normas Regulamentadoras de Pesquisas Envolvendo Seres Humanos. Diário Oficial da União 1996; 16 out.

19. Cesar JA, Oliveira-Filho JA, Bess G, Cegielka R, Machado J, Gonçalves TS, Neumann NA. Perfil dos idosos residentes em dois municípios pobres das regiões Norte e Nordeste do Brasil: resultados de estudo transversal de base populacional. Cad Saude Publica 2008; 24(8):1835-1845.

20. Pagotto V, Nakatani AYK, Silveira, EA. Fatores associados à autoavaliação de saúde ruim em idosos usuários do Sistema Único de Saúde. Cad Saude Publica 2011; 27(8):1593-1602.

21. Duarte YAO, Lebrão ML, Lima FD. Contribuição dos arranjos domiciliares para o suprimento de demandas assistenciais dos idosos com comprometimento funcional em São Paulo, Brasil. Rev Panam Salud Pública 2005; 17(5/6):370-378.

22. Lebrão ML, Duarte YAO. O projeto Sabe no município de São Paulo: uma abordagem inicial. 1a Edição. Brasília: Organização Pan-Americana da Saúde; 2003.

23. Costa SV, Ceolim MF, Neri AL. Problemas de sono e suporte social: estudo multicêntrico Fragilidade em Idosos Brasileiros. Rev Latino-Am Enfermagem 2011; 19(4):920-927.

24. Camarano AA. Envelhecimento da população brasileira: uma contribuição demográfica. In: Freitas EV, Cançado FAX, Gorzoni ML, organizadores. Tratado de geriatria e gerontologia. 2a Edição. Rio de Janeiro: Guanabara Koogan; 2006.

25. Fuhrer R, Dufouil C, Antonucci TC, Shipley MJ, Helmer C, Dartigues JF. Psychological disorder and mortality in French older adults: do social relations modify the association? Am J Epidemiol 1999; 149(2):116-126.

26. Cornwell B, Schumm LP, Laumann EO, Graber J. Social Networks in the NSHAP Study: Rationale, Measurement, and Preliminary Findings. J Gerontol B Psychol Sci Soc Sci 2009; 64B(Supl. 1):i47-i55.

27. Avlund K, Damsgaard MT, Holstein BE. Social relations and mortality. An eleven year follow-up study of 70-year-old men and women in Denmark. Soc Sci Med 1998; 47(5):635-643.

28. Guedea MTD, Albuquerque FJB, Tróccoli BT, Noriega JAV, Seabra MAB, Guedea RLD. Relação do bem-estar subjetivo, estratégias de enfrentamento e apoio social em idosos. Psicol Reflex Crit 2006; 19(2):301-308.

29. Due P, Holstein B, Lund R, Modvig J, Avlund K. Social relations: Network, support and relational strain. Soc Sci Med 1999; 48(5):661-673.
30. Alvarenga MRM, Oliveira MAC, Domingues MAR, Amendola F, Faccenda O. Rede de suporte social do idoso atendido por equipes de Saúde da Família. Cien Saude Colet 2011; 16(5):2603-2611.

31. Freiheit EA, Hogan DB, Strain LA, Schmaltz HN, Patten SB, Eliasziw M, Maxwell CJ. Operationalizing frailty among older residents of assisted living facilities. BMC Geriatr 2011; 11:23.

32. Santos-Eggimann B, Cuénoud P, Spagnoli J, Junod J. Prevalence of frailty in middle-aged and older community-dwelling Europeans living in 10 countries. J Gerontol A Biol Sci Med Sci 2009; 64(6):675681.

33. Espinoza SE, Jung I, Hazuda H. Lower Frailty Incidence Among Mexican American than Among European American Older Adults: The San Antonio Longitudinal Study of Aging. J Am Geriatr Soc 2010; 58(11):2142-2148.

34. Alvarado BE, Zunzunegui MV, Béland F, Bamvita JM. Life course social and health conditions linked to frailty in Latin American older men and women. J Gerontol A Biol Sci Med Sci 2008; 63(12):1399-1406.

35. Graham JE, Snih SA, Berges IM, Ray LA, Markides KS, Ottenbacher KJ. Frailty and 10-year mortality in community-living Mexican American older adults. Gerontology 2009; 55(6):644-651.

36. Garcia-Garcia FJ, Gutierrez Avila G, Alfaro-Acha A, Amor Andres MS, De Los Angeles De La Torre Lanza M, Escribano Aparicio MV, Humanes Aparicio S, Larrion Zugasti JL, Gomez-Serranillo Reus M, Rodriguez-Artalejo F, Rodriguez-Manas L; Toledo Study Group. The prevalence of frailty syndrome in an older population from Spain. The Toledo Study for Healthy Aging. J Nutr Health Aging 2011; 15(10):852-856.

37. Costa TB, Neri AL. Medidas de atividade física e fragilidade em idosos: dados do Fibra Campinas, São Paulo, Brasil. Cad Saude Publica 2011; 27(8):15371550.

38. Syddall HE, Roberts HC, Evandrou M, Cooper C, Bergman H, Sayer AA. Prevalence and correlates of frailty among community-dwelling older men and women: findings from the Hertfordshire Cohort Study. Age and Ageing 2010; 39(2):197-203.

39. Cawthon PM, Marshall LM, Michael Y, Dam TT, Ensrud KE, Barrett-Connor E, Orwoll ES; Osteoporotic Fractures in Men Research Group. Frailty in older men: prevalence, progression, and relationship with mortality. J Am Geriatr Soc 2007; 55(8): 1216-1223.

40. Panes VCB. Adaptação dos componentes da síndrome de fragilidade e avaliação da fragilização dos idosos residentes no município de São Paulo: estudo SABE saúde, bem-estar e envelhecimento [dissertação]. São Paulo (SP): Universidade de São Paulo; 2010.

41. Avila-Funes JA, Helme C, Amieva H, BarbergerGateau P, Le Goff M, Ritchie K, Portet F, Carrière I, Tavernier B, Gutiérrez-Robledo LM, Dartigues JF. Frailty among community-dwelling elderly people in France: the three-city study. J Gerontol A Biol Sci Med Sci 2008; 63(10):1089-1096. 
42. Fried LP, Ferrucci L, Darer J, Williamson JD, Anderson G. Untangling the concepts of disability, frailty, and comorbidity: implications for improved targeting and care. J Gerontol A Biol Sci Med Sci 2004; 59(3):255-263.

43. Lima-Costa MF, Firmo JOA, Uchôa E. A estrutura da auto-avaliação da saúde entre idosos: projeto Bambuí. Rev Saude Publica 2004; 38(6):827-834.

44. Andrew MK, Mitnitski AB, Rockwood K. Social Vulnerability, Frailty and Mortality in Elderly People. PLoS One 2008; 3(5):e2232.

45. Cohen S, Wills TA. Stress, social support, and the buffering hypothesis. Psychol Bull 1985; 98(2):310357.

Artigo apresentado em 15/02/2012

Aprovado em 01/03/2012

Versão final apresentada em 05/03/2012 